BMJ Open

Diabetes

Research

\& Care

\title{
Quantifying dermal microcirculatory changes of neuropathic and neuroischemic diabetic foot ulcers using spatial frequency domain imaging: a shade of things to come?
}

\author{
Grant A Murphy (D) , ${ }^{1}$ Rajinder P Singh-Moon, ${ }^{2}$ Amaan Mazhar, ${ }^{2}$ David J Cuccia, ${ }^{2}$ \\ Vincent L Rowe, ${ }^{1}$ David G Armstrong ${ }^{1}$
}

To cite: Murphy GA, Singh-Moon RP, Mazhar A, et al. Quantifying dermal microcirculatory changes of neuropathic and neuroischemic diabetic foot ulcers using spatial frequency domain imaging: a shade of things to come? BMJ Open Diab Res Care 2020;8:e001815. doi:10.1136/ bmjdrc-2020-001815

Received 4 August 2020 Revised 6 October 2020 Accepted 3 November 2020
A) Check for updates

(C) Author(s) (or their employer(s)) 2020. Re-use permitted under CC BY-NC. No commercial re-use. See rights and permissions. Published by BMJ.

${ }^{1}$ Department of Surgery, University of Southern California Keck School of Medicine, Los Angeles, California, USA

${ }^{2}$ Department of Research and Development, Modulim, Irvine, California, USA

Correspondence to Grant A Murphy; gamurphy@usc.edu

\section{ABSTRACT}

Introduction The use of non-invasive vascular and perfusion diagnostics are an important part of assessing lower extremity ulceration and amputation risk in patients with diabetes mellitus. Methods for detecting impaired microvascular vasodilatory function in patients with diabetes may have the potential to identify sites at risk of ulceration prior to clinically identifiable signs. Spatial frequency domain imaging (SFDI) uses patterned nearinfrared and visible light spectroscopy to determine tissue oxygen saturation and hemoglobin distribution within the superficial and deep dermis, showing distinct microcirculatory and oxygenation changes that occur prior to neuropathic and neuroischemic ulceration.

Research designs and methods 35 patients with diabetes mellitus and a history of diabetic foot ulceration were recruited for monthly imaging with SFDI. Two patients who ulcerated during the year-long longitudinal study were selected for presentation of their clinical course alongside the dermal microcirculation biomarkers from SFDI.

Results Patient 1 developed a neuropathic ulcer portended by a focal increase in tissue oxygen saturation and decrease in superficial papillary hemoglobin concentration 3 months prior. Patient 2 developed bilateral neuroischemic ulcers showing decreased tissue oxygen saturation and increased superficial papillary and deep dermal reticular hemoglobin concentrations.

Conclusions Wounds of different etiology show unique dermal microcirculatory changes prior to gross ulceration. Before predictive models can be developed from SFDI, biomarker data must be correlated with the clinical course of patients who ulcerate while being followed longitudinally.

Trial registration number NCT03341559.

\section{INTRODUCTION}

Diabetes mellitus (DM) is associated with diminished sensation and compromised perfusion in the lower extremities, substantially increasing the risk of foot complications, such as ulcers. Approximately one-third of patients with DM will develop a diabetic foot ulcer over the course of their lifetime. ${ }^{1}$ Along

\section{Significance of this study}

What is already known about this subject?

- The use of lower extremity perfusion assessment is an important part of the diagnostic workup for diabetic foot ulcers, as these measures can indicate whether a patient has the capacity to heal following ulceration.

What are the new findings?

- Spatial frequency domain imaging (SFDI) is a noninvasive imaging method that shows unique dermal microcirculation and oxygenation changes in preulcerative and ulcerative tissue that differentiates neuropathic and neuroischemic etiology in patients with diabetes mellitus; this appears to be the first manuscript in the medical literature to specifically identify these patterns.

How might these results change the focus of research or clinical practice?

- Predictive modeling developed from SFDI image analysis may be able to identify tissue at risk of ulceration before clinical signs are present, enabling earlier and more successful use of focused prophylactic treatment against foot ulcers.

with degrading quality of life, ulcers often lead to severe infection and amputation, driving disproportionate healthcare costs in patients with $\mathrm{DM}^{2}{ }^{2}$ These complications are further exacerbated by high reulceration rates (up to $40 \%$ in 1 year), likely perpetuated by unresolved underlying conditions. ${ }^{1}$ As a result, ulcer prevention has become a primary strategy for managing diabetic foot diseases. ${ }^{3-6}$ This is why lower extremity complications of DM now constitute a top 10 global disability burden based on WHO data. ${ }^{78}$

Numerous studies have elucidated contributing factors that predict ulcer occurrence 
in patients with DM, one of which is the presence of vascular compromise such as peripheral artery disease (PAD). ${ }^{19-11}$ Non-invasive vascular and perfusion diagnostics are routinely used for assessing the presence of PAD, ${ }^{1}{ }^{12}{ }^{13}$ however, these tests rely on bulk measurements often taken some anatomic distance away from the site of ulceration. This is especially true for wounds on the plantar aspect of the foot, the most common site of ulcer presentation. Thus, it is difficult to infer from these measurements the location and severity of plantar microvascular dysfunction which may herald ulcerative signatures. Prior studies examining plantar tissue circulation suggest that regional changes could be used to identify early signatures of at-risk sites for ulceration. ${ }^{10} 14$ Although encouraging, the data are limited and the relationship between ulcer etiology and dermal perfusion signatures remain unclear.

To this end, these case presentations aim to examine pre-ulcerative changes in dermal microcirculation and oxygenation for ulcers of different etiology using spatial frequency domain imaging (SFDI). SFDI is a non-contact, optical imaging technology that can directly assess circulation in the skin by measuring hemoglobin $(\mathrm{Hb})$ distribution and oxygenation. ${ }^{15}$ SFDI has been demonstrated in a number of applications to quantify circulationincluding flap perfusion and burn assessment. ${ }^{16}{ }^{17}$ SFDI was also compared with current methods of non-invasive vascular assessment (ie, ankle-brachial and toe-brachial indexes and waveforms) in patients at risk for PAD and showed that hemoglobin distribution between layers was a sensitive metric to identify patients with PAD with DM. ${ }^{18}$ More recently, SFDI was used to assess ulcer risk and prognosticate ulcer onset in patients with DM. ${ }^{14}$ Comparing SFDI signatures in patients with DM with and without a history of ulceration, the former were reported to exhibit significantly lower hemoglobin in the papillary dermis and higher dermal tissue oxygen saturation consistent with arterio-venous (AV) shunting observations previously reported. ${ }^{18}$ A limitation of this study was that a single image time point was used to relate to ulcer development outcomes within a year follow-up. In this work, case studies of longitudinal SFDI monitoring are presented for neuropathic and neuroischemic instances, highlighting peri-ulcer circulatory changes throughout the wound cycle.

\section{RESEARCH DESIGN AND METHODS} Study design

A total of 35 patients were recruited for this observational study, with inclusion criteria consisting of patients with previous or current diabetic foot ulcers. Patients with heart failure or rheumatoid arthritis were excluded from the observational study. Inclusion criteria for case presentation included new ulceration during the study, with at least one pre-ulcerative SFDI image obtained. To ensure baseline comparisons with contralateral foot regions, patients with previous forefoot or digit amputation were excluded for case presentation; patients with Charcot neuroarthropathy were also excluded. Written consent was obtained for each patient allowing longitudinal collection of SFDI imaging data in concert with medical histories and treatment information. Subjects underwent plantar imaging at the start of normal visits every 4-6 weeks for 1 year. Additionally, foot temperatures, vessel palpability, visible callus presence and visible wound presence observations were recorded at each visit. Two patients with initially intact forefeet who ulcerated during the course of the study were selected for presentation and clinically demonstrated different etiologies, one neuropathic (patient 1) and the other neuroischemic (patient 2). Due to the bilateral ulcer presentations in patient 2 , the left foot will be referenced as $2 \mathrm{a}$ and the right foot as $2 b$.

\section{SFDI imaging}

A commercial SFDI system (Research RS, Modulim, Irvine, California, USA) was used to obtain plantar circulation biomarker maps. A combination of visible and near-infrared light, as well as planar and patterned illumination, is used to interrogate both shallow and deep (up to $3 \mathrm{~mm}$ ) tissue volumes (figure 1A). Images of tissue reflected light intensities are then processed to render three two-dimensional circulation biomarker maps (figure 1B): tissue oxygen saturation $\left(\mathrm{StO}_{2}\right)$, total superficial papillary hemoglobin $\left(\mathrm{HbT}_{1}\right)$ and total subsurface reticular hemoglobin $\left(\mathrm{HbT}_{2}\right)$. The total imaging time was $<5$ min per patient including device calibration, patient foot positioning, instrument alignment, SFDI data acquisition and processing, and parametric map display.

\section{Wound classification}

We used the Society for Vascular Surgery Wound, Ischemia, Foot Infection (WIfI) system to score limb threat at each visit. WIfI is a widely validated measure of limb threat that scores each characteristic as 'none, mild, moderate or severe' from 0 to $3 .^{19-22}$

\section{RESULTS}

\section{Patient 1: neuropathic ulcer}

Patient 1 presented in their early 60 s with poorly controlled type $2 \mathrm{DM}$ (hemoglobin A1c 9.3\% at 28 weeks) and peripheral neuropathy, along with other signs suggestive of hyperglycemia-related organ damage, including microproteinuria.

At week 0 , patient 1 presented to the clinic with calluses along the interphalangeal joint of the left hallux (figure 2A). A small right hallux laceration with signs of healing was present, along with calluses on the plantar surface below the right second metatarsal phalangeal joint (MTPJ). Palpation of lower extremity arteries revealed strong pulses. SFDI biomarkers showed focal areas of elevated dermal oxygen saturation and reduced papillary (superficial) hemoglobin at the second MTPJ bilaterally, although to a greater extent on the right foot. Compared with forefoot medians, right second MTPJ 


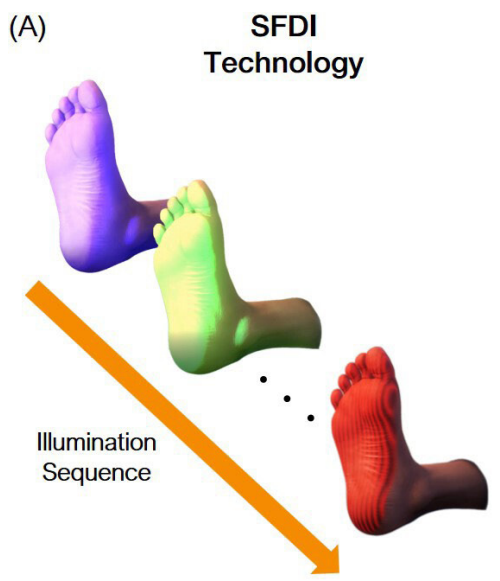

(B) $\begin{gathered}\text { SFDI } \\ \text { Biomarkers }\end{gathered}$

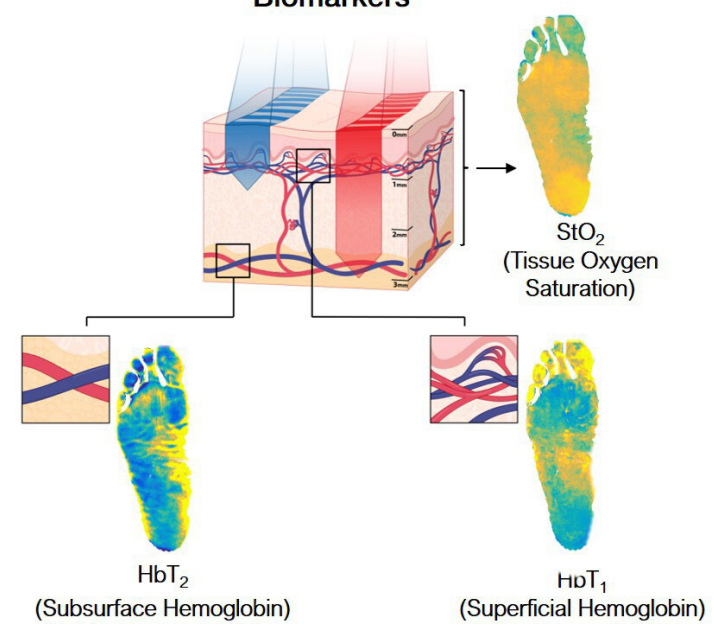

Figure 1 Spatial frequency domain imaging (SFDI) technology for plantar foot imaging. (A) SFDI uses a combination of visible and near-infrared light to interrogate tissue and recover functional circulation characteristics. (B) Diagram illustrating SFDI characterization of tissue oxygen saturation $\left(\mathrm{StO}_{2}\right)$ and stratified hemoglobin content $\left(\mathrm{HbT}_{1}\right.$ and $\left.\mathrm{HbT}_{2}\right)$. $\mathrm{HbT}_{1}$ represents total hemoglobin levels originating from superficial microvasculature while $\mathrm{HbT}_{2}$ represents the subsurface macrovascular total hemoglobin levels.

regional averages were $16.7 \%$ higher for $\mathrm{StO}_{2}$ and $50 \%$ lower for $\mathrm{HbT}_{1}$; in the left foot, $\mathrm{StO}_{2}$ and $\mathrm{HbT}_{1}$ regional averages were $10.5 \%$ higher and $30.7 \%$ lower, respectively.

Thirteen weeks later, the patient presented with a superficial $1.0 \times 1.0 \mathrm{~cm}$ wound at the right second MTPJ, secondary to a flexible hammertoe with retrograde buckling (figure 2B). Images showed elevated oxygenation and subsurface hemoglobin within the peri-ulcer region. The wound and undermining callus were debrided, cleaned with dilute hypochlorous acid and covered with
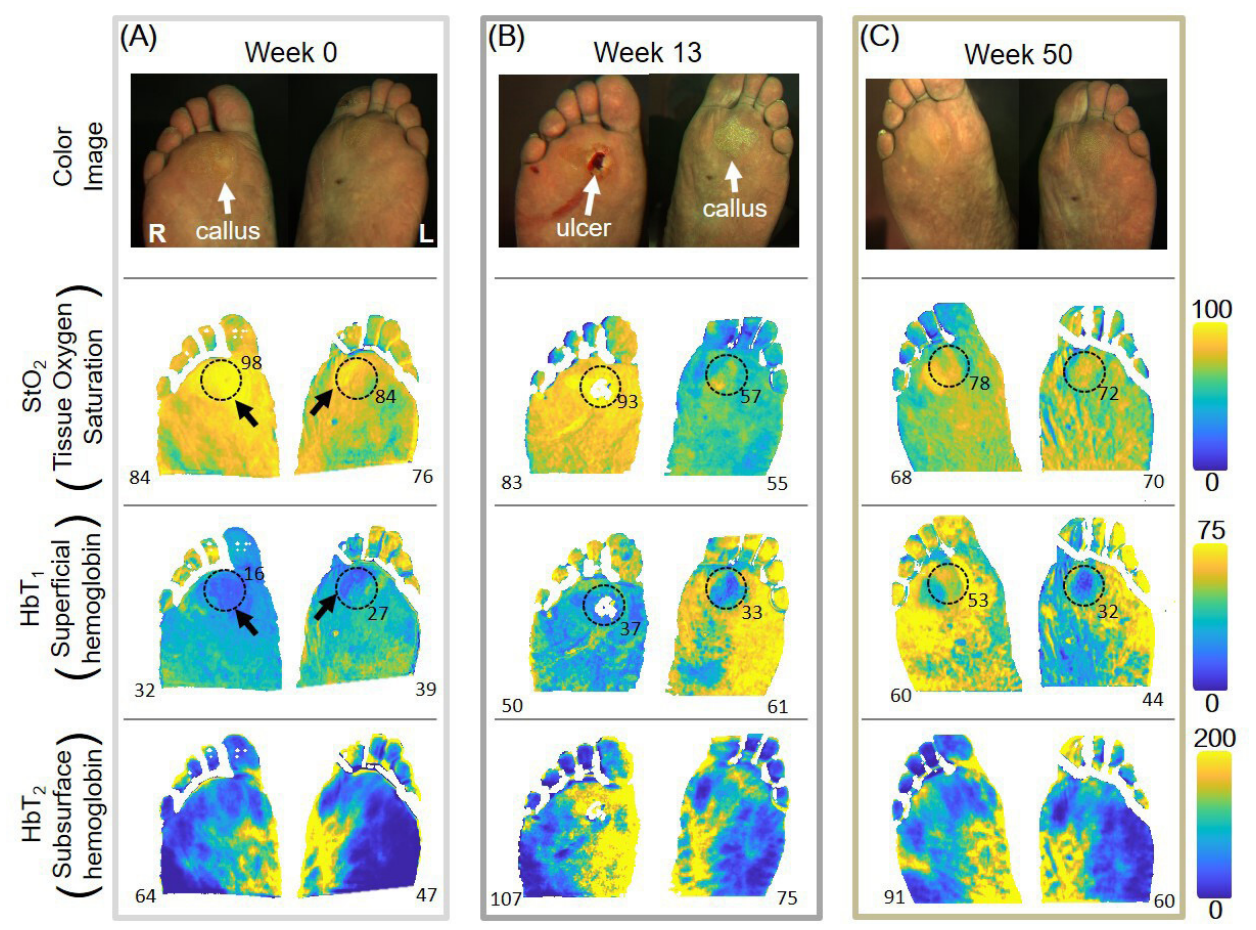

Figure 2 Longitudinal microcirculatory monitoring for patient 1. Spatial frequency domain imaging (SFDI) biomarker profiles of the plantar forefoot are shown at weeks 0 (A), 13 (B) and 50 (C). Visual inspection of color images at week 0 show a seemingly innocuous appearance, however focal areas of elevated $\mathrm{StO}_{2}$ and decreased $\mathrm{HbT}_{1}$ at the second metatarsal phalangeal joint (MTPJ) bilaterally suggest a latent issue. At wound presentation (right second MTPJ) 13 weeks later, $\mathrm{StO}_{2}$ remains elevated within the peri-wound region coupled with rising $\mathrm{HbT}_{1}$. At week 50 on visible healing postosteotomy, $\mathrm{StO}_{2}$ and $\mathrm{HbT}_{1}$ signatures exhibited reduced focal activity near the right second MTPJ with a shift toward the third MTPJ. Numbers at the base of each foot represent forefoot median values. Numbers near dashed circles represent median values over the circumscribed region. 
silver and foam dressings; the patient was offloaded in a 'healing sandal'. On the contralateral foot, a minor callus formed under the second MTPJ, which coincided with a site of focally reduced papillary hemoglobin and increased $\mathrm{StO}_{2}$ at week 0 .

At week 16 , the $1.0 \times 1.0 \mathrm{~cm}$ MTPJ wound was debrided again and the patient was placed in a total contact cast (TCC). At week 28, the patient presented with cellulitis of the right foot secondary to a superficial infection at the second MTPJ ulcer $(2.0 \times 1.0 \mathrm{~cm})$. The right forefoot demonstrated edema and erythema without signs of deep tissue infection. The patient began empiric oral antibiotics (clindamycin and ciprofloxacin) and was placed in a removable cast boot for offloading.

Five weeks later, the patient had complete resolution of the cellulitis; the next week, a right second metatarsal osteotomy and wound debridement were performed.

Postoperative follow-up at week 34 revealed a noninfected wound with immature epithelial coverage and residual callus under the right second MTPJ. Two weeks later, the surgical site was fully healed and the callus was noted to have decreased in area and depth. At week 50, SFDI showed normalization of $\mathrm{StO}_{2}$ and $\mathrm{HbT}_{1}$ levels at the right second MTPJ where prior osteotomy was performed (figure 2C) and a shift in focal behavior toward the third MTPJ. The patient would go on to develop calluses of the left plantar third digit and left dorsolateral fifth proximal interphalangeal joint without ulceration.

\section{Patient 2: neuroischemic ulcers}

Patient 2 presented in their late 50s with type $2 \mathrm{DM}$ and severe PAD. This patient presented to the clinic after being offered an above-knee amputation for a chronic left heel ulcer, as severe PAD led to concerns whether the patient could heal a below-knee amputation. Several weeks prior to enrollment in this study, the patient had a right toe brachial index (TBI) of 0.19 and a left TBI of 0.00 .

The patient underwent surgical treatments for the heel ulcer, with a left partial calcanectomy and wound debridement performed 1 week into the study and a superficial circumflex iliac artery perforator (SCIP) flap reconstruction 4 weeks into the study. At week 9, bilateral erythema presented at the hallux tips and on the left first MTPJ. SFDI biomarker maps showed substantial elevation of hemoglobin levels across both papillary and reticular layers in the hallux compared with the remaining forefoot, bilaterally (figures $3 \mathrm{~A}$ and $4 \mathrm{~A}$ ). In the right
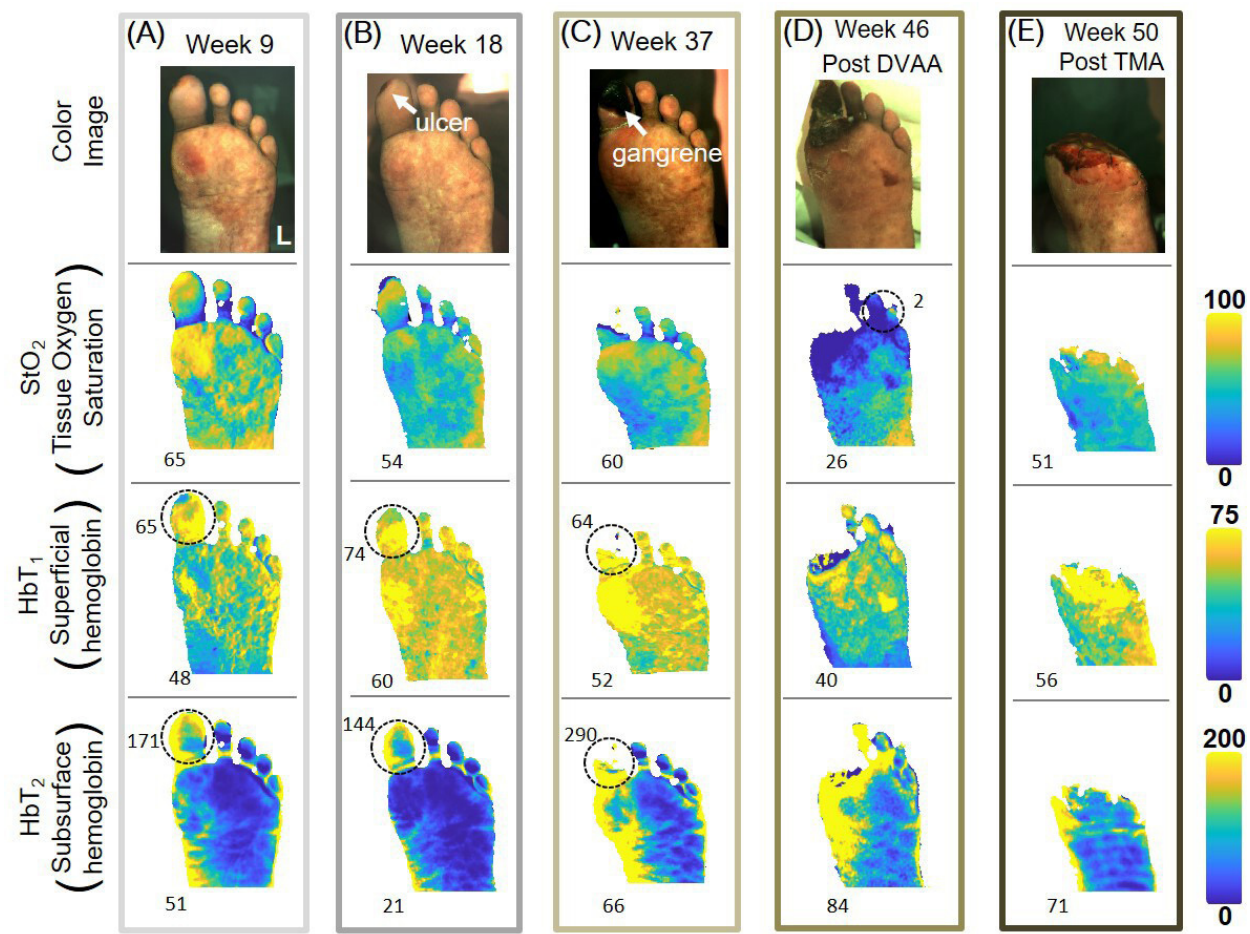

Figure 3 Longitudinal microcirculatory monitoring for patient 2 (left foot). Spatial frequency domain imaging (SFDI) biomarker profiles of the plantar forefoot are shown at weeks 9 (A), 18 (B), 37 (C), 46 (D) and 50 (E). At week 9, the hallux exhibited general signs of hyperemia and blood pooling as indicated by superficial $\left(\mathrm{HbT}_{1}\right)$ and subsurface hemoglobin $\left(\mathrm{HbT}_{2}\right)$, respectively. The pre-ulcer redness at the hallux tip coincided with focally elevated $\mathrm{StO}_{2}$ and reduced $\mathrm{HbT}_{1}$. Signatures of hyperemia and pooling persisted at week 18 throughout hallux ischemic wound presentation. Gangrene presentation at week 37 showed poor oxygenation surrounding the wound site and progressive hyperemia and pooling in the subhallux region. At week 46 following left popliteal artery to DVAA bypass, the eventually spreading of gangrene to the medial forefoot and lesser digits was forecasted by substantially low $\mathrm{StO}_{2}$ in these regions. At week 50,2 weeks following TMA, signs of circulatory restoration were observed as indicated by a re-normalization of $\mathrm{StO}_{2}$ and $\mathrm{HbT}_{2}$ values. Numbers at the base of each foot represent forefoot median values. DVAA, dorsal venous arch arterialization; TMA, transmetatarsal amputation. 

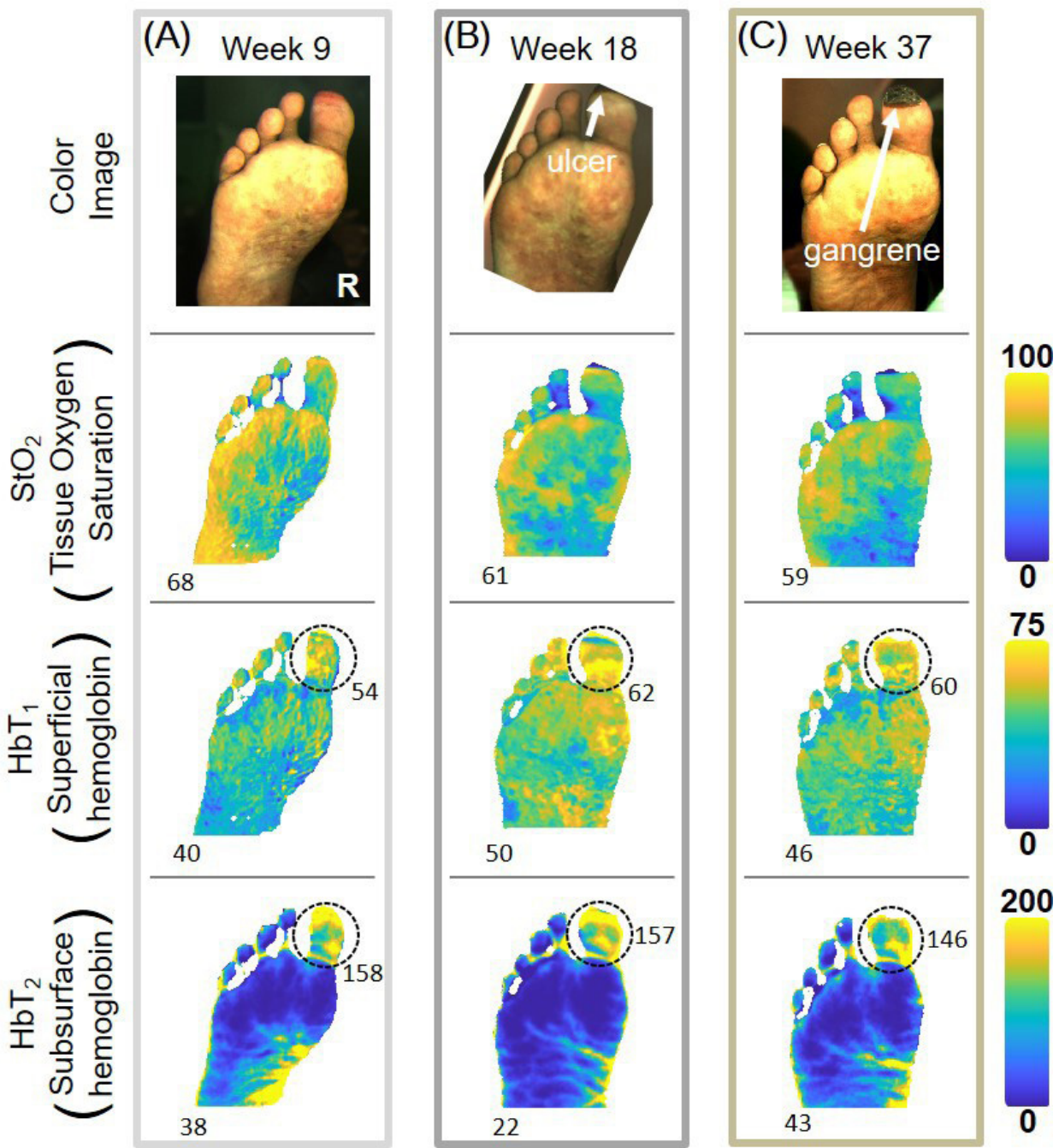

Figure 4 Longitudinal microcirculatory monitoring for patient 2 (right foot). Spatial frequency domain imaging (SFDI) biomarker profiles of the plantar forefoot are shown at weeks 9 (A), 18 (B) and 37 (C). At week 9, redness at the hallux tip coincided with hyperemia and pooling of blood as indicated by superficial $\left(\mathrm{HbT}_{1}\right)$ and subsurface hemoglobin $\left(\mathrm{HbT}_{2}\right)$, respectively. General hallux signatures of hyperemia and pooling persisted at week 18 throughout ischemic wound presentation coupled with substantially low oxygenation $\left(\mathrm{StO}_{2}\right)$ localized at the tip. The peri-wound region demonstrated elevated $\mathrm{StO}_{2}$ and reduced $\mathrm{HbT}_{1}$. With gangrenous wound progression at week 37 , an extension of the poorly oxygenated region was observed with further elevation of $\mathrm{HbT}_{1}$ and $\mathrm{HbT}_{2}$. Numbers at the base of each foot represent forefoot median values. Numbers near dashed circles represent median values over the circumscribed region.

foot, hallux $\mathrm{HbT}_{1}$ and $\mathrm{HbT}_{2}$ values were $35 \%$ and $416 \%$ greater than forefoot medians; in the left foot, hallux values were at $35 \%$ and $335 \%$ greater.

At week 18, the patient presented with bilateral ischemic hallux wounds. Similar hemoglobin signatures persisted, along with concomitant low oxygenation localized to the wound site (figures 3B and 4B). By week 35, these hallux wounds had worsened and the patient began topical $\mathrm{O}_{2}$ therapy; necrotic tissue was debrided. During follow-up at week 37 , worsening bilateral gangrene was noted along with osteomyelitis of the left lower extremity (LLE) (figures 3C and 4C). Visible areas of necrosis directly coincided with severely low $\mathrm{StO}_{2}(<5)$. The peri-gangrenous area exhibited sustained elevated hallux hemoglobin levels compared with forefoot medians; on the left foot,
$\mathrm{HbT}_{1}$ and $\mathrm{HbT}_{2}$ were $23 \%$ and $439 \%$ greater, respectively; the right side comparisons were $30 \%$ and $335 \%$ greater.

At week 39, the patient presented to the emergency department with ulceration, erythema and purulent drainage of the LLE. Arterial duplex of the LLE showed severe arterial wall calcification of the popliteal, extending to the tibial-peroneal trunk, with monophasic flow in the anterior tibial (AT) and multiphasic flow in the posterior tibial (PT). Radiographic findings were suggestive of osteomyelitis and the patient began intravenous ceftriaxone and vancomycin; the patient was discharged 4 days later.

At 43 weeks, the patient underwent a left popliteal artery to dorsal venous arch arterialization (DVAA); toe pressures were $0 \mathrm{~mm} \mathrm{Hg}$ bilaterally. The right hallux 
gangrene remained stable and well demarcated, having changed minimally over the past 9 weeks.

At 46 weeks, the patient returned to the emergency department for pain and swelling of the LLE; a balloon angioplasty of the DVAA was performed. Figure 3D shows SFDI biomarkers prior to angioplasty. The forefoot appeared oxygen depleted, particularly along the lesser digits and medial forefoot regions within the $\mathrm{StO}_{2}$ map. These areas coincided with increased $\mathrm{HbT}_{1}$ and $\mathrm{HbT}_{2}$. At week 47, gangrene had spread to the forefoot and the second and third digits. At week 48, a transmetatarsal amputation (TMA) was performed and covered with a wound vacuum. The following week, the TMA wound was debrided and covered with an acellular matrix. Figure 3E shows SFDI biomarkers 1 week following debridement and covering. Images showed resolution of both poor oxygenation and pooled reticular hemoglobin.

\section{CONCLUSION}

In patients with DM, chronic lower extremity wounds can generally be categorized as either neuropathic or neuroischemic. Wounds can be further stratified using the four factors of the WIfI threatened limb classification system: wound, ischemia, foot infection and function. ${ }^{212}$ Wounds arising in patients with peripheral neuropathy without signs of vascular disease are considered neuropathic, as loss of protective sensation and disruption of autonomic function are the primary causes of ulceration. In patients with vascular disease, reduced blood flow leads to necrosis of tissue in poorly perfused angiosomes. While patients with DM can develop purely ischemic wounds, chronic ulceration is most often neuropathic or neuroischemic, as purely ischemic wounds are relatively rare. ${ }^{23}$ These two patients were selected because their medical histories and clinical course are consistent with the classic presentation of neuropathic (1) and neuroischemic (2) wounds.

SFDI biomarkers provide an insight to the differing pathological processes of these two types of wounds. Previous studies have shown baseline perfusion differences between patients with DM and patients without $\mathrm{DM}$, as well as statistically significant differences between PAD patients with and without DM. ${ }^{18}$ These differences are likely related to the microangiopathy and autonomic neuropathy seen in DM. As early as 91 days prior to the ulceration of patient 1's right second MTPJ, a relative increase in $\mathrm{StO}_{2}$ and decrease in $\mathrm{HbT}_{1}$ was observed when compared with the contralateral foot's corresponding region of interest-a finding consistent with the previous studies showing significant differences in both the global baseline and ulcer-preceding elevations of $\mathrm{StO}_{2}$ in patients with DM. ${ }^{14} 18$ This localized pattern of covariation (elevated $\mathrm{StO}_{2}$ and recessed $\mathrm{HbT}_{1}$ ) seems to signal at-risk sites due to mechanical stresses (ie, high plantar pressure and repetitive trauma) that precipitate pre-ulcer lesions and skin breakdown. This explanation is further supported by callus development at the left second MTPJ at week 13 following a similar, but less pronounced, focal $\mathrm{StO}_{2}$ and $\mathrm{HbT}_{1}$ appearance. Another corroborating detail is the anatomical shift in $\mathrm{StO}_{2}$ and $\mathrm{HbT}_{1}$ signatures over to the adjacent third MTPJ following right second MTPJ osteotomy, which may indicate plantar stress redistribution.

$\mathrm{StO}_{2}$ represents the ratio between oxy-Hb and total $\mathrm{Hb}$ (oxy-Hb+deoxy-Hb) ${ }^{24}$ Therefore, an increased $\mathrm{StO}_{2}$ in the presence of decreased $\mathrm{HbT}_{1}$ serves as a marker of dermal microcirculation dysfunction within the papillary dermis. Capillary basement membrane thickening from chronic hyperglycemia may reduce $\mathrm{O}_{2}$ diffusion into the surrounding tissue ${ }^{25}$ thus causing a relative decrease in deoxy- $\mathrm{Hb}$ and consequentially elevating $\mathrm{StO}_{2}$. Another contributing factor to reduced deoxy-Hb is autonomic neuropathy, where loss of vasomotor tone on the dermal AV shunts allows blood to bypass the papillary plexus. ${ }^{26}{ }^{27}$ The highest concentration of these dermal AV shunts are on the palmar and plantar surfaces of the hands and feet ${ }^{28}$; physiologically, autonomic regulation of these shunts allows the sympathetic nervous system to promote heat loss by increasing superficial dermal blood flow. Pathologically, this likely reduces the transport of $\mathrm{O}_{2}$ and nutrients to the dermal regenerative cells, inhibiting wound healing and exacerbating neuropathy by reducing perfusion of the vasa nervorum. ${ }^{28}$

Changes in microvascular function are well documented in patients with neuropathic foot ulcers. SFDI could potentially be used as a tool for functional assessment of microcirculatory changes, as patients with diabetes with foot ulcers demonstrate an impaired skin vasodilatory response to pressure, acetylcholine administration, lower extremity heating and ischemia-induced reactive hyperemia when compared with non-ulcer patients with diabetes and healthy controls. ${ }^{29}{ }^{30}$ Given the notable differences in vasodilatory response between ulcer and non-ulcer patients with diabetes, the ability of SFDI to detect impaired microcirculatory responses to pressure and heat should be tested, as this may be an effective way of identifying patients at risk of ulceration.

The neuroischemic perfusion signatures from patient 2 show a different pre-ulcer microcirculatory environment to that of patient 1: elevated $\mathrm{HbT}_{1}$ and $\mathrm{HbT}_{2}$ at the eventual wound site and surrounding area. These features were apparent as early as 9 weeks prior to ulceration. On visible wound presentation, there is low tissue oxygenation; thus, the coinciding elevated $\mathrm{HbT}_{1}$ may indicate an accumulation of deoxy-Hb within the superficial dermis. While patient 1 had minimal differences in $\mathrm{HbT}_{2}$ between corresponding regions of interest, ulcers $2 \mathrm{a}$ and 2b both demonstrated increased $\mathrm{HbT}_{2}$ when compared with global foot perfusion. The combination of low $\mathrm{StO}_{2}$ with increased $\mathrm{HbT}_{1}$ and $\mathrm{HbT}_{2}$ may signify pooling of deoxy-Hb in the dermal microcirculation.

For patients with DM, neuroischemic wounds are more commonly found at the foot margins, while neuropathic ulcers tend to form on the plantar surface. ${ }^{31}$ In $2 \mathrm{a}$, there were focal increases in $\mathrm{StO}_{2}$ and decreases in $\mathrm{HbT}_{1}$ at 
the initial pre-ulcerative sites on the distal hallux, briefly displaying signatures more consistent with neuropathic ulceration. Since the wound location and patient history is more consistent with a neuroischemic etiology, the transient perfusion changes may be indicative of an acute inflammatory response to tissue necrosis. Moreover, it is conceivable that the local circulation behavior in these cases is a function of the relative severity of PAD, neuropathy and their complex interaction within a given patient. Additional studies are required to investigate the contributory relationship between these component causes and SFDI biomarkers.

It is possible that iatrogenic causes contributed to the amputation of $2 \mathrm{a}$. The addition of a highly vascularized SCIP flap to the patient's heel may have induced a vascular steal syndrome in the forefoot, as low-pressure vessels in the forefoot were unable to compensate when a parallel circuit (ie, flap) was added. With monophasic flow in the AT and a toe pressure of $0 \mathrm{~mm} \mathrm{Hg}$, the patient was at risk for ischemic complications following reconstructive surgery. The patient's forefoot had minimal collateral circulation, as there was bilateral hypoplasia of the PT, incomplete plantar arterial arches and minimal runoff into the medial plantar arteries on angiography. During the SCIP flap reconstruction, extensive calcification of the PT was noted and the flap was anastomosed with the artery in an end-side fashion; intraoperative flap assessment and follow-up indicated adequate arterial blood supply and venous drainage of the flap. While the treatment of the calcaneal ulcer was successful, 2a had far more tissue loss than 2b during this study. Perfusion changes following reconstructive surgery showed a precipitous drop in $\mathrm{StO}_{2}$ at the hallux and second digit, although global LLE changes were minimal for all biomarkers.

The DVAA was performed since a venous conduit was the only revascularization target; DVAA is reserved as a last resort treatment in limb salvage when severe PAD precludes bypassing to an arterial target, with complications like amputation being common following intervention. ${ }^{32}$ The gangrene rapidly worsened following the DVAA and a TMA was performed about 1 month later. Post-DVAA but pre-TMA, $\mathrm{StO}_{2}$ decreased and $\mathrm{HbT}_{2}$ increased rapidly; $\mathrm{HbT}_{1}$ changed minimally. These perfusion changes may be necrosis-related rather than a result of the venous arterialization, as the $\mathrm{StO}_{2}$ reduction and increased $\mathrm{HbT}_{2}$ seem to precede gross necrotic changes in areas associated with ischemia. This suggests SFDI circulation monitoring could potentially be used as an anatomical delimiting tool in strategizing amputations.

While recent studies using SFDI have attempted to develop statistical models for identifying high-risk preulcerative perfusion signatures,${ }^{14}$ classification and analysis of wounds by specific etiology has not been used. Since neuropathic and neuroischemic wounds can be differentiated clinically and occur in patients with particular comorbidities, subset analysis of SFDI data should divide wounds by clinical categorization to differentiate the dermal microcirculatory signals associated with the two most common DM-related wound etiologies. Although these cases demonstrate the proposed pathophysiological model of neuropathic and neuroischemic diabetic foot ulcers, there are inherent limitations given the small number of wounds presented. Going forward, the full dataset of SFDI data from the observational study will be analyzed, stratifying wounds by etiology and time (pre-ulcer, ulcerative, post-ulcer). Wounds with similar etiology and timing will be compared with contralateral controls, if present, and the differences will be pooled to demonstrate if there are statistically significant differences between etiology and time points throughout the healing process. Once larger SFDI datasets have been collected, stratified and analyzed, this technology can begin being tested as an ulcer-prevention tool, with physicians using the dermal microcirculation biomarkers to guide earlier prophylactic measures against lower extremity ulceration.

Acknowledgements The authors would like to thank Karen D'Huyvetter for her support in coordinating the study and data collection.

Contributors AM, DJC and DGA were involved in study conceptualization and design. GAM, RPS-M, AM and DGA were involved in data curation, analysis and interpretation. GAM and RPS-M wrote the original manuscript. GAM, RPS-M, AM, DJC, VLR and DGA critically reviewed and edited the manuscript. AM and DJC obtained funding and study resources. All authors have read and approved the final manuscript.

Funding This study was supported by National Institutes of Health/National Institute of Diabetes and Digestive and Kidney Diseases (NIH/NIDDK) grant 2R44DK094625.

Competing interests RPS-M, AM and DJC are full-time employees of Modulim and have financial interests in the company. Modulim is commercializing SFDI technology.

Patient consent for publication Obtained.

Ethics approval This study was approved by the Institutional Review Board of Keck Medical Center of the University of Southern California (HS-17-00926).

Provenance and peer review Not commissioned; externally peer reviewed.

Data availability statement All data relevant to the study are included in the article. Requests for deidentified biomarker data can be sent to info@modulim.com and may be used with consent from Modulim.

Open access This is an open access article distributed in accordance with the Creative Commons Attribution Non Commercial (CC BY-NC 4.0) license, which permits others to distribute, remix, adapt, build upon this work non-commercially, and license their derivative works on different terms, provided the original work is properly cited, appropriate credit is given, any changes made indicated, and the use is non-commercial. See: http://creativecommons.org/licenses/by-nc/4.0/.

\section{ORCID iD}

Grant A Murphy http://orcid.org/0000-0002-9775-9325

\section{REFERENCES}

1 Armstrong DG, Boulton AJM, Bus SA. Diabetic foot ulcers and their recurrence. N Engl J Med 2017;376:2367-75.

2 Driver VR, Fabbi M, Lavery LA, et al. The costs of diabetic foot: the economic case for the limb salvage team. J Vasc Surg 2010;52:17S-22.

3 Armstrong DG, Lavery LA, Vela SA, et al. Choosing a practical screening instrument to identify patients at risk for diabetic foot ulceration. Arch Intern Med 1998;158:289-92.

4 Boyko EJ, Ahroni JH, Cohen V, et al. Prediction of diabetic foot ulcer occurrence using commonly available clinical information: the Seattle diabetic foot study. Diabetes Care 2006;29:1202-7. 
5 Lavery LA, Armstrong DG, Vela SA, et al. Practical criteria for screening patients at high risk for diabetic foot ulceration. Arch Intern Med 1998;158:157-62.

6 McGill M, Molyneaux L, Yue DK. Which diabetic patients should receive podiatry care? an objective analysis. Intern Med J 2005;35:451-6.

7 Lazzarini PA, Pacella RE, Armstrong DG, et al. Diabetes-Related lower-extremity complications are a leading cause of the global burden of disability. Diabet Med 201810.1111/dme.13680. [Epub ahead of print: 23 May 2018].

8 Zhang Y, Lazzarini PA, McPhail SM, et al. Global disability burdens of diabetes-related lower-extremity complications in 1990 and 2016. Diabetes Care 2020;43:964-74.

9 Waaijman R, de Haart M, Arts MLJ, et al. Risk factors for plantar foot ulcer recurrence in neuropathic diabetic patients. Diabetes Care 2014;37:1697-705.

10 Yudovsky D, Nouvong A, Schomacker K, et al. Assessing diabetic foot ulcer development risk with hyperspectral tissue oximetry. $J$ Biomed Opt 2011;16:026009.

11 Saiko G, Lombardi P, Au Y, et al. Hyperspectral imaging in wound care: a systematic review. Int Wound J 2020:1-17.

12 Beckman JA, Duncan MS, Damrauer SM, et al. Microvascular disease, peripheral artery disease, and amputation. Circulation 2019;140:449-58.

13 Monteiro-Soares M, Boyko EJ, Ribeiro J, et al. Predictive factors for diabetic foot ulceration: a systematic review. Diabetes Metab Res Rev 2012;28:574-600.

14 Lee S, Mey L, Szymanska AF, et al. SFDI biomarkers provide a quantitative ulcer risk metric and can be used to predict diabetic foot ulcer onset. J Diabetes Complications 2020;34:107624.

15 Gioux S, Mazhar A, Cuccia DJ. Spatial frequency domain imaging in 2019: principles, applications, and perspectives. J Biomed Opt 2019;24:1-18.

16 Nguyen JT, Lin SJ, Tobias AM, et al. A novel pilot study using spatial frequency domain imaging to assess oxygenation of perforator flaps during reconstructive breast surgery. Ann Plast Surg 2013;71:308-15.

17 Ponticorvo A, Rowland R, Baldado M, et al. Evaluating clinical observation versus spatial frequency domain imaging (SFDI), laser speckle imaging (LSI) and thermal imaging for the assessment of burn depth. Burns 2019;45:450-60.

18 Weinkauf C, Mazhar A, Vaishnav K, et al. Near-instant noninvasive optical imaging of tissue perfusion for vascular assessment. $J$ Vasc Surg 2019;69:555-62.

19 Armstrong DG, Mills JL. Juggling risk to reduce amputations: the three-ring Circus of infection, ischemia and tissue loss-dominant conditions. Wound Medicine 2013;1:13-14.
20 Conte MS, Bradbury AW, Kolh P, et al. Global vascular guidelines on the management of chronic limb-threatening ischemia. Eur $J$ Vasc Endovasc Surg 2019;58:e33:S1-109.

21 Mills JL, Conte MS, Armstrong DG, et al. The Society for vascular surgery lower extremity threatened limb classification system: risk stratification based on wound, ischemia, and foot infection (WIfl). $J$ Vasc Surg 2014;59:e1-2:220-34.

22 Khan T, Plotkin A, Magee GA, et al. Functional ambulatory status as a potential adjunctive decision-making tool following wound, level of ischemia, and severity of foot infection assessment. J Vasc Surg 2020;72:738-46.

23 Oyibo SO, Jude EB, Tarawneh I, et al. A comparison of two diabetic foot ulcer classification systems: the wagner and the University of Texas wound classification systems. Diabetes Care 2001;24:84-8.

24 Sowa MG, Kuo W-C, Ko AC-T, et al. Review of near-infrared methods for wound assessment. J Biomed Opt 2016;21:091304.

25 Boyd RB, Burke JP, Atkin J, et al. Significance of capillary basement membrane changes in diabetes mellitus. J Am Podiatr Med Assoc 1990;80:307-13

26 Lefrandt JD, Bosma E, Oomen PHN, et al. Sympathetic mediated vasomotion and skin capillary permeability in diabetic patients with peripheral neuropathy. Diabetologia 2003;46:40-7.

27 Ngo BT, Hayes KD, DiMiao DJ, et al. Manifestations of cutaneous diabetic microangiopathy. Am J Clin Dermatol 2005;6:225-37.

28 Van Dam PS, Cotter MA, Bravenboer B, et al. Pathogenesis of diabetic neuropathy: focus on neurovascular mechanisms. Eur $J$ Pharmacol 2013;719:180-6.

29 Vouillarmet J, Josset-Lamaugarny A, Michon P, et al. Neurovascular response to pressure in patients with diabetic foot ulcer. Diabetes 2019;68:db180694-836.

30 Argarini R, McLaughlin RA, Joseph SZ, et al. Visualizing and quantifying cutaneous microvascular reactivity in humans by use of optical coherence tomography: impaired dilator function in diabetes. Am J Physiol Endocrinol Metab 2020;319:E923-31.

31 Pitei DL, Lord M, Foster A, et al. Plantar pressures are elevated in the neuroischemic and the neuropathic diabetic foot. Diabetes Care 1999;22:1966-70.

32 Houlind K, Christensen J, Hallenberg C, et al. Early results from an angiosome-directed open surgical technique for venous arterialization in patients with critical lower limb ischemia. Diabet Foot Ankle 2013;4. doi:10.3402/dfa.v4i0.22713. [Epub ahead of print: 17 Dec 2013].

33 Rowe VL, Hood DB, Lipham J, et al. Initial experience with dorsa venous arch arterialization for limb salvage. Ann Vasc Surg 2002;16:187-92. 